bildung zwar sinnvoll. Angesichts des komplexen analytischen Ansatzes erscheint diese dichotomische Gegenüberstellung jedoch zumindest ausbaufähig. Im Sinne einer weiteren Theoriebildung wäre darüber nachzudenken, wie diese Typologie weiter ausdifferenziert werden könnte, um die Eigenheiten einer größeren Zahl an empirischen Fällen besser abzubilden und den internationalen Vergleich so zu erleichtern. Damit ändert sich jedoch nichts an der Gesamtbewertung: Es liegt eine anspruchsvolle, empirisch gesättigte, theoretisch und methodisch reflektierte, sehr gut lesbare und innovative Studie vor, die für die weitere politikwissenschaftliche Forschung zur strategischen Regierungssteuerung Maßstäbe setzt.

Henrik Gast

\title{
Parlamentarische Kontrolle von Militäroperationen - Verfahren im Vergleich ohne Berücksichtigung des Informellen
}

\author{
Ondarza, Nicolai von: Legitimatoren ohne Einfluss? Nationale Parlamente in Entscheidungspro- \\ zessen zu militärischen EU- und VN-Operationen im Vergleich, Nomos Verlagsgesellschaft, \\ Baden-Baden 2012, 363 Seiten, $€ 59$,-.
}

Der Strukturwandel der internationalen Beziehungen seit dem Ende des Kalten Krieges hat die Bedingungen für die nationale Außen- und Sicherheitspolitik grundlegend verändert. Dazu gehört das Phänomen der zunehmenden multinationalen Militäroperationen zur weltweiten Konfliktlinderung. Diese im Kontext internationaler Organisationen durchgeführten Missionen können gleichwohl nur stattfinden, sofern deren Mitgliedstaaten freiwillig bestimmte Kontingente ihrer nationalen Streitkräfte entsenden. Doch diese Form der Beteiligung an internationalen Einsätzen stellt die politischen Institutionen und Verfahren auf nationaler Ebene vor neue Herausforderungen: Wie kommen die Entscheidungen in der Praxis zustande? Und wie ist es um deren parlamentarische Kontrolle und demokratische Legitimation bestellt?

In den vergangenen Jahren sind bereits erste Studien erschienen, die die Mechanismen in Deutschland näher betrachten. ${ }^{1}$ Vergleichende Analysen sind indes rar. Diese Lücke füllt Nicolai von Ondarza. Mit seiner Dissertation strebt er nicht weniger an als das systematische Vermessen parlamentarischer Beteiligungsrechte an sicherheitspolitischen Entscheidungen in den europäischen Nationalstaaten. Sein Urteil fällt klar aus: Zwar existiert eine parlamentarische Kontrolle und Mitwirkung in unterschiedlichen Formen - gemessen an der Tragweite der Entscheidungen sei sie aber überall zu gering.

Da die Bandbreite der Verfahren und formellen Beteiligungsrechte in den nationalen Parlamenten erheblich ist, konzentriert sich der Autor auf eine Auswahl von fünf Staaten.

1 Vgl. Nina Philippi, Bundeswehr-Auslandseinsätze als außen- und sicherheitspolitisches Problem des geeinten Deutschland, Frankfurt am Main 1997; Markus Wölfle, Die Auslandseinsätze der Bundeswehr und ihre Auswirkungen auf die Rolle Deutschlands im internationalen System, Bonn 2005; Daniel Sigloch, Auslandseinsätze der deutschen Bundeswehr: verfassungsrechtliche Möglichkeiten und Grenzen, Hamburg 2006; Andreas M. Rauch, Auslandseinsätze der Bundeswehr, Baden-Baden 2006; Dieter Wiefelspütz, Auslandseinsätze der Bundeswehr und der Bundestag, in: ZParl, 39. Jg. (2008), H. 2, S. $203-218$. 
Mithilfe der Kategorien „Stellung des Parlaments im politischen System“, „sicherheitspolitische Orientierung“ und „parlamentarische Rechte in der Verteidigungspolitik“ gelingt es ihm souverän, Deutschland, Frankreich, Irland, Polen und Spanien als geeignete Fallauswahl mit hinreichender Varianz in den Indikatoren herauszuarbeiten. Die beiden Militäroperationen EUFOR RD CONGO sowie UNIFIL vor der Küste Libanons (beide 2006) dienen dabei als Fallstudien, um die Entscheidungsverfahren sichtbar zu machen - und gleichzeitig zu überprüfen, ob Einsätze im Kontext von EU beziehungsweise VN auf nationaler Ebene unterschiedlich behandelt werden.

Im Vergleich zeigt sich klar: Bei der Entscheidungsvorbereitung gibt es keine unmittelbare Beteiligung der nationalen Parlamente. Gleichwohl verfügen diese - jeweils auf ihre eigene Art - im Laufe der Entscheidungsfindung über eine Reihe formeller Kontrollrechte, die Ondarza in einem Analyseraster systematisiert und auf einer fünfstufigen Skala vergleichend bewertet. Dabei wird deutlich, dass der „Zugang zu Informationen“, die „Mitentscheidungsrechte“, die „Haushaltsrechte“ sowie die „Präferenzen der parlamentarischen Akteure zur GSVP“ in den betrachteten Ländern unterschiedlich stark ausgeprägt sind. Die Zusammenfassung in Tabellen verschafft dem Leser dabei einen angenehm schnellen Überblick.

In der Analyse sieht Ondarza die rechtlichen Vorgaben zur verpflichtenden parlamentarischen Zustimmung, wie sie in Deutschland (Parlamentsbeteiligungsgesetz), Irland und neuerdings auch in Spanien existieren, als entscheidenden Faktor für das Kontrollpotential. Die in den Parlamenten vorherrschenden normativen sicherheitspolitischen Einstellungen und Orientierungen hingegen lassen nicht erkennen, ob und wie intensiv parlamentarische Beteiligungsrechte tatsächlich ausgeübt werden. Es besteht aber ein grundsätzlicher Zielkonflikt: In beiden untersuchten Operationen haben die Regierungen auf internationaler und europäischer Ebene vorab die Beteiligung ihres Landes zugesagt und erst anschließend die parlamentarischen Zustimmungsverfahren eingeleitet. Damit stünden „die von einem effektiven multilateralen Konfliktmanagement benötigten frühen Zusagen seitens der Regierungen [...] im Konflikt mit der Anforderung nach tatsächlicher parlamentarischer Beteiligung" (S. 297).

Um den diagnostizierten Mangel an Mitwirkung zu mindern, plädiert Ondarza in seinen abschließenden Handlungsempfehlungen unter anderem dafür, dem Europäischen Parlament bei EU-geführten Militäroperationen mehr Kontrollrechte und Mitwirkungsmöglichkeiten einzuräumen - und seine Abgeordneten in einem Pfad zu bestärken, den sie in der Sicherheitspolitik ohnehin schon eingeschlagen haben. ${ }^{2}$ Außerdem soll eine stärker verknüpfte interparlamentarische Kooperation die genauere Kontrolle intergouvernementaler Politik gewährleisten.

Zwar stellt Ondarza richtigerweise die Verbindung her, dass die spätere Zustimmungspflicht vorab zu einer stärkeren informellen Einbindung der Parlamentarier geführt hat. Aber insgesamt misst er den informellen Kontroll- und Mitwirkungsmöglichkeiten ${ }^{3}$ zu wenig

2 Vgl. Julian Böcker, Demokratiedefizit der Sicherheits- und Verteidigungspolitik der EU? Analyse des deutschen, britischen und Europäischen Parlaments, Baden-Baden 2012.

3 Vgl. Manfred Schwarzmeier, Parlamentarische Mitsteuerung. Strukturen und Prozesse informalen Einflusses im Deutschen Bundestag, Wiesbaden 2001; Sabine Kropp, Regieren in Parlamentarismus und Parteiendemokratie. Informale Institutionen als Handlungsressource für Exekutive und Parlament, in: Werner Jann / Klaus König (Hrsg.), Regieren zu Beginn des 21. Jahrhunderts, Tübingen 2008, S. 49 - 86; René Lüddecke, Parlamentarisierung der nationalen Außenpolitik, BadenBaden 2010. 
Bedeutung bei. Außerdem erscheinen die Parlamente zu sehr als einheitliche Akteure. Die funktionslogische Trennung von Regierung und Opposition mit ihren unterschiedlichen Anreizen zu Mitwirkung, Kontrolle oder wohlwollender Begleitung des Regierungshandelns, die sich aus der jeweils spezifischen dynamischen Beziehung zwischen Exekutive und Legislative eröffnet ${ }^{4}$, bleibt weitgehend unbeachtet. Wünschenswert wären auch mehr Präzision und Tiefe bei Begriffsbestimmungen der unterschiedlichen Facetten von Kontrolle und Mitwirkung gewesen. ${ }^{5}$

Insgesamt gelingt Ondarza in seiner Dissertation aber ein guter exemplarischer, empirisch reichhaltiger und übersichtlich strukturierter Einblick in die Entscheidungsverfahren zur Entsendung von Streitkräften in multilaterale Missionen. Der Vergleich der Einbindungsmuster von fünf nationalen Parlamenten enthüllt jedoch nur den offen sichtbaren Teil ihrer Rolle als Träger der demokratischen Legitimation dieser sensiblen sicherheitspolitischen Entscheidungen. Die darunter liegenden Schichten informeller Kontroll- und Mitwirkungsmöglichkeiten werden zu wenig freigelegt.

Auf diese Weise bestätigt sich die im Titel aufgeworfene Frage als selbsterfüllende Prophezeiung: Parlamente blieben somit in der Sicherheitspolitik trotz allmählicher Verbesserung vorerst doch „bloße Notare anderweitig getroffener Entscheidungen “6 und damit „Legitimatoren ohne (großen) Einfluss“. Ein differenzierteres Bild würde entstehen, wenn man die parlamentarische Funktionslogik von Regierung und Opposition systematisch betrachten - und damit informelle Kanäle und Strukturen der parlamentarischen Mitwirkung aufspüren würde.

René Lüddecke

4 Vgl. unter anderem Everhard Holtmann / Werner J. Patzelt (Hrsg.), Kampf der Gewalten? Parlamentarische Regierungskontrolle - gouvernementale Parlamentskontrolle, Wiesbaden 2004.

5 Siehe bereits bei Winfried Steffani, Gewaltenteilung und Parteien im Wandel, Wiesbaden 1997; Richard G. Mulgan, Holding Power to Account. Accountability in Modern Democracies, Basingstoke 2003.

6 Jean Blondel, Comparative Legislatures, Englewood Cliffs 1973, S. 22.

\section{Europapolitische Mitwirkung des Bundestags - präzise Beschreibung mit konzeptionellen Schwächen}

Schulz, Fabian: Die Mitwirkung des Deutschen Bundestags in europäischen Angelegenheiten. Politikgestaltung im Spannungsfeld von Europäisierung und Entparlamentarisierung, Waxmann Verlag, Münster 2011, 312 Seiten, €29,90.

Wie gestalten sich die Beteiligungsrechte des Deutschen Bundestags in europäischen Angelegenheiten, und auf welchen Wegen findet parlamentarische Mitwirkung tatsächlich statt? Diese Fragen bilden den Ausgangspunkt der von Fabian Schulz vorgelegten Arbeit zur europapolitischen Mitwirkung des Bundestags. Der analytische Fokus der erklärtermaßen deskriptiven Studie liegt auf den "tatsächlich praktizierten Mitwirkungswegen“ (S. 12). Diese können, wie Schulz hervorhebt, sowohl auf formalen als auch auf informalen Beteili- 